Math. Model. Nat. Phenom.

Vol. 4, No. 3, 2009, pp. 1-11

DOI: $10.1051 / \mathrm{mmnp} / 20094301$

\title{
Multiphase and Multiscale Trends in Cancer Modelling
}

\author{
L. Preziosi ${ }^{1}$, A. Tosin \\ Department of Mathematics, Politecnico di Torino, Italy
}

\begin{abstract}
While drawing a link between the papers contained in this issue and those present in a previous one (Vol. 2, Issue 3), this introductory article aims at putting in evidence some trends and challenges on cancer modelling, especially related to the development of multiphase and multiscale models.
\end{abstract}

Key words: cancer modelling, multiscale models, hybrid models, multiphase systems. AMS subject classification: 92C10, 92B05, 92C17

\section{Introduction}

Comparing the recent literature with that developed ten years ago, it can be realized that not only has the attention of the research community toward cancer modelling increased considerably (the number of papers per year published on the subject increased four times), but also the type of models developed have significantly evolved.

In our opinion, this is due on one hand to the introduction of new types of modelling frameworks, e.g., multiphase models operating at the tissue scale and several types of Individual Cell-Based Models (IBMs) operating at the cellular scale, and on the other hand to the increased attention paid to the genomical and proteomical aspects related to tumour growth.

One of the trends appearing in the literature, which characterizes the present and the previous special issue of this journal (Vol. 2, Issue 3), is the necessity to take into account phenomena occurring at different spatial and/or temporal scales. This means, for instance,

\footnotetext{
${ }^{1}$ Corresponding author. E-mail: luigi.preziosi@polito.it
} 
trying to insert in IBMs or in continuous models the details of the molecular mechanisms which take place within the cell and govern cell motion, duplication, death.

The aim of this article is then to outline such trends. Without claiming to be exhaustive (to achieve this we refer the reader to $[9,16,60]$ ), the following sections will briefly review multiphase, nested, and hybrid models, though the distinction among them is not so well defined.

\section{Multiphase models}

Multiphase models were mainly developed under the simple observation that tumours are made of several constituents. This change of viewpoint allowed not only to describe the density variation within the tumour and the host tissue, but also to evaluate the evolution of stresses and of the interstitial pressure, and to link the mechanics of tumours to their behaviour and growth.

After the first bi-phasic models, the trend now is to develop multiphase models considering that tumours, like many other tissues, are made of at least three constituents, i.e., cells, extracellular matrix, and extracellular liquid. An example of these models is [13], which focusses on the growth of tumour cords along capillaries. Other examples are $[10,11,12,19,22,31,47]$. Recently, Lowengrub and coworkers [26, 62] developed a model in which an adhesion energy is introduced to describe the adhesion properties of cellular populations. Still working in a multiphase framework, the final model consists of a fourth-order nonlinear advection-reaction-diffusion equation of Cahn-Hilliard type, characterized by the regularisation of an adhesion term that could otherwise lead to an ill-posed problem.

The effect of mechanical and environmental cues on the behaviour and growth of tumours and tissues is currently widely studied. Chaplain et al. [22] developed a model accounting for contact inhibition of growth, and showed how a misperception of the compression state of the tissue can generate by itself a clonal advantage on the surrounding cells, leading ultimately to the replacement and the invasion of the healthy tissue by the tumour. Also [33, 38, 48] looked at this problem, using individual cell-based models. As we will see in Section 5, this led to a useful comparison between the two modelling approaches.

Another aspect that is attracting more and more attention is a proper description of the mechanical characteristics of tumours. Indeed, most papers tend to treat tumours as liquids, which is of course a simplification. In some cases, it is important to confer a solid-like behaviour to the ensemble of tumour cells. Some non trivial theoretical difficulties arise in doing that. In fact, cells duplicate and die, the tumour grows, the extracellular matrix (ECM) and the external environment are continuously remodelled, and tumour cells deform and undergo an internal re-organisation even in absence of growth, which might also be related to the detachment of metastases. In order to account for these phenomena, it is necessary to revisit some of the classical and confortable concepts of continuum mechanics, such as the meaning of reference configuration and of deformation. Indeed, unlike inert matter, in principle it is not clear which reference should be used to measure deformation s of a growing living tissue, because the material is continuously changing. This problem was tackled in 
$[2,3]$ by applying the concept of evolving natural configurations, which consists in splitting the evolution in growth and elastic deformation. Subsequently, this approach was generalised in [5] to the multiphase framework, accounting also for the internal re-organisation and the deformation of the ECM. In this case, the natural configuration evolves because of two different contributions: growth and cell re-organisation, the latter being related to the experimental observation that adhesion bonds between cells have a finite strength and might break and build up during the evolution. Hence, the macroscopic model uses information from the microscopic scale, a procedure that will be discussed in the following section.

\section{Nested Models}

It is clear that tumour growth and invasion depend on the behaviour of the single cells and on the interactions they have with the environment. The latter depend in turn on several chemical cues, e.g., genetic information, gene expression, activation of particular signalling pathways. Consequently, even if one is interested only in describing a biological phenomenon from the macroscopic point of view, it becomes natural and fundamental to include into the models at the tissue or cellular scale also the processes occurring at the sub-cellular scale, e.g., the activation of specific protein cascades.

For this reason, some models developed to describe the growth of tumour masses are nowadays paying more and more attention to the chemical phenomena inside the cell, nesting in the macroscopic (or mesoscopic) representation one or more modules accounting for processes at the microscopic scale. Thanks to the most recent discoveries in the field of genomics, proteomics, and system biology, these descriptions are expanding considerably, and, in our opinion, their use will increase considerably.

In this respect, models at the cellular scale, such as IBMs, seem more flexible and suited to include sub-cellular mechanisms. We refer the reader, for instance, to $[49,50]$, where the detailed dynamics of E-cadherin/ $\beta$-catenin interactions is incorporated to obtain a deeper description of cell-cell adhesion, or, in this issue, to [18] that focusses on the MAP-kinase regulatory network and, in particular, on the interplay between Ras and Fas determining selfrenewal and differentiation of erythroid progenitors. Alarcon and coworkers $[1,45]$ include in their cellular automata for vascularised tumours some aspects related to the VEGF and the p53 patways, in addition to the proteins governing the cell cycle.

A wonderful overview of these methodology is given in $[7,8]$. In particular, [8] summarises the characteristics of what in $[35,36]$ it is called evolutionary hybrid cellular automata model (and we prefer here to classify as nested model). In this approach, the evolution of each single cell is accompanied by a micro-environment response network. The latter is modelled using a feed-forward artificial neural network, that determines the behaviour of the cells from the input obtained by the environmental variables through the internal genotype and phenotype.

The same paper also discusses the model developed by Rejniak and coworkers $[51,52,53]$, who used an immerse boundary method to model each single tumour cell. This model also 
includes discrete adhesion forces exerted on the cell membrane, as well as contractile forces used to describe cell mitosis.

In a spirit similar to [35, 36], Jiang et al. [39] used a cellular Potts model to describe the growth of an avascular multicellular spheroid, where the behaviour of each single tumour cell is determined by a Boolean network which regulates the expression of some key proteins controlling the cell cycle.

In both approaches, the evolution of nutrients is treated by means of a reaction-diffusion equation. For this reason, the model has some hybrid characteristics (see the next section).

In some structured population models of cancer, which is the main topic of this issue, the evolution along the cell cycle is related to the activation of specific signalling pathways, or to the internal molecular content, e.g., DNA (see also, in this issue, the paper by Friedman [32] and the review by Clarimbault [25], where more references can be found). In particular, in [27] the transitions through the restriction point is related to the presence of specific control proteins, e.g., cyclin/CDK complexes. In the same framework, some papers [54, 55] describe the efficacy of several chemotherapeutic drugs.

When using continuous models, the conceptual jump is bigger, because the parameters appearing in the partial differential equations (PDEs) need to be related more closely to the sub-cellular mechanisms. For instance, regarding the growth terms, in [22] the activation of mechano-transduction pathways and its relation with the phenomenon of contact inhibition of growth is taken into account, and in $[12,13,17,61]$ a detailed description of the metabolism, both aerobic and anaerobic, is given. Regarding instead the mechanical behaviour of tumour spheroids, $[5,46,47]$ try to relate the parameters in the constitutive model of the tumour mass to the measurements performed at the cellular scale. Specifically, they focus on the strength of integrin-mediated adhesion bonds connecting the cell to the ECM, and on that of cadherin-mediated bonds acting among the cells. However, the link between macroscopic and microscopic scales obtained in this way is often more qualitative than quantitative. One should probably use deeper mathematical methods, such as homogenisation techniques or other fluid-dynamic and diffusion limits to get more formal and consistent results (see, for instance, $[23,24,37,41,56,57]$, that however sometimes deal with different biological applications).

\section{Hybrid Models}

First of all, it need be stressed that in the literature there are two types of models generally called hybrid. The most common one, and historically the first, uses a discrete approach (e.g., discretization of PDEs, cellular automata, IBMs) for some constituents and a continuous approach for other constituents (e.g., diffusion equations or more general partial differential equations). Typically, cells are described using spatially discrete variables, while molecules and ECM are described using spatially continuous variables. Recently, a second type of models have been introduced, in which some particular constituents (usually the cells) are described by means of either discrete variables in a time-dependent portion of the 
spatial domain or continuous variables in the rest of the domain. These models, that we call interfacing hybrid models, will be briefly discussed in the next section.

Standard examples of hybrid models are some IBMs in which the diffusion of nutrients through the boundary of a multicellular spheroid is related to the duplication/death of cells (see, for instance, $[29,30]$ ).

A brief review of different hybrid models stemming from the seminal work by Anderson and Chaplain [6] can be found in [7, 8] (where the reader can also find most of the references by the research group on the topic). There, the equation for the evolution of the tip cells of the capillaries is discretized, giving rise to what is called a hybrid discrete-continuous model, that allows to describe the structure of the capillary network in a very efficient way. The evolution of this equation depends on the diffusion and uptake of several chemical factors promoting angiogenesis and migration.

The model was then enhanced to include on the one hand the remodelling of the network and the transport of chemotherapeutic drugs $[43,44,58]$, and on the other hand the coupling with a growing tumour, the remodelling of the ECM, and the production of matrix degrading enzymes $[42,63]$. In this last case, the tumour mass is assumed to have a constant density and its expansion is governed by a potential flow giving rise to a Darcy's-like closure [4]. The ECM is rigid and remodelling, whereas matrix degrading enzymes, tumour angiogenic factors, and nutrients diffuse.

Still dealing with angiogenesis, in [14] a cellular Potts model is instead used to describe the cells composing the capillaries. The evolution of chemical factors is again linked to proper reaction-diffusion equations.

\section{Interfacing (Hybrid) Models}

As it might be easily guessed, there is not a single way to approach the same biological phenomenon and, in some cases, the same biological problem can be studied using different models. Of course, each approach has its advantages and disadvantages. For instance, PDE models are more suited and computationally cheaper to describe the behaviour of tissues from the macroscopic point of view, but, as discussed above, it is more difficult to account for sub-cellular mechanisms such as signal transduction, expression or internalisation of receptors, and so on. Conversely, IBMs can focus more closely on the cellular level, but they might become computationally expensive when using a large number of cells, and inefficient in providing a general outlook on the system as a whole.

Luckily, it is often not necessary to keep the same level of detail throughout the tissue. The idea behind what we call interfacing hybrid models is to use the modelling tools like a microscope, focussing on the cellular or sub-cellular level where and when needed and blurring when such a detailed description is not needed. In $[40,59]$ the spatial domain is partitioned in few time-dependent domains, and in each piece of this moving puzzle a different modelling framework is used, e.g. IBM and continuous models, so that it is possible to take advantage of the positive aspects of both frameworks. 
The first step in this process is to compare, for the same biological phenomenon, the results obtained via different models. This was done, for instance, for the growth of avascular colonies in [20, 34] where an IBM and a multiphase model were used. This way, it is possible to support the upscaling process and get an idea of the relationship between those parameters that cannot be easily linked.

The next step would be to get the mechanical behaviour of non-growing IBM tissues from standard virtual mechanical tests, e.g., stress relaxation, creep and indentation tests, steady shear, and cyclic loading and deformations. In our opinion, this might be done by building in silico experiments, like those performed in rheology, for instance using cone-and-plate rheometers. Ensembles of cells with some given microscopic characteristics (e.g., cell-cell adhesion or cell compressibility) could then be put in these virtual experiments and tested. One could get as output the macroscopic parameters to be used in the constitutive equation of the multiphase model, that hopefully guarantee the preservation of the mechanical properties when switching back and forth from IBMs to macroscopic models.

All this must be supported by suitable mathematical procedures that allow models to cross-talk. Besides classical limits linking kinetic to fluid-dynamic models $[41,15,21]$ and the homogenisation methods mentioned above, Drasdo [28] introduced a coarse-graining method that provides a link between different scales (IBMs and continuous models) and biophysical processes.

We expect big developments in this field, considering that other research directions deserve to be investigated. For instance, one could exploit the experience accumulated in numerical analysis on ovelapping domain decomposition methods to build overlapping hybrid models, based on the construction of a patchwork of non necessarily disjoint subdomains.

\section{Acknowledgements}

A. Tosin acknowledges the support of the National Institute for Advanced Mathematics (INdAM) and the "Compagnia di San Paolo" foundation.

\section{References}

[1] T. Alarcon, H. M. Byrne, P. K. Maini. A multiple scale model for tumor growth. Multiscale Model. and Simul., 3 (2005), 440.

[2] D. Ambrosi, F. Mollica. On the mechanics of a growing tumor. Internat. J. Engrg. Sci., 40 (2002), 1297-1316.

[3] D. Ambrosi, F. Mollica. The role of stress in the growth of a multicell spheroid. J. Math. Biol., 48 (2004), 477-499.

[4] D. Ambrosi, L. Preziosi. On the closure of mass balance models for tumor growth. Math. Models Methods Appl. Sci., 12 (2002), 737-754. 
[5] D. Ambrosi, L. Preziosi. Cell adhesion mechanisms and stress relaxation in the mechanics of tumours. Biomech. Model. Mechanobiol. doi:10.1007/s10237-008-0145-y.

[6] A. R. A. Anderson, M. A. J. Chaplain. Continuous and discrete mathematical models of tumor-induced angiogenesis. Bull. Math. Biol., 60 (1998), 857-899.

[7] A. R. A. Anderson, M. A. J. Chaplain, K. A. Rejniak. Single-cell-based models in biology and medicine. Springer (2007).

[8] A. R. A. Anderson, K. A. Rejniak, P. Gerlee, V. Quaranta. Modelling of cancer growth, evolution and invasion: bridging scales and models. Math. Model. Nat. Phenom., 2 (2007), 1-29.

[9] R. P. Araujo, D. L. S. McElwain. A history of the study of solid tumour growth: the contribution of mathematical modelling. Bull. Math. Biol., 66 (2004), 1039-1091.

[10] R. P. Araujo, D. L. S. McElwain. A mixture theory for the genesis of residual stresses in growing tissues. I. A general formulation. SIAM J. Appl. Math., 65 (2005), 1261-1284.

[11] R. P. Araujo, D. L. S. McElwain. A mixture theory for the genesis of residual stresses in growing tissues. II. Solutions to the biphasic equations for a multicell spheroid. SIAM J. Appl. Math., 66 (2005), 447-467.

[12] S. Astanin, L. Preziosi. Mathematical modelling of the Warburg effect in tumour cords. J. Theor. Biol., 258 (2009), 578-590.

[13] S. Astanin, A. Tosin. Mathematical model of tumour cord growth along the source of nutrient. Math. Model. Nat. Phenom., 2 (2007), 153-177.

[14] A. L. Bauer, T. L. Jackson, Y. Jiang. A cell-based model exhibiting branching and anastomosis during tumor-induced angiogenesis. Biophys. J., 92 (2007), 3105-3121.

[15] N. Bellomo, A. Bellouquid, J. Nieto, J. Soler, P. K. Maini. Multicellular biological growing systems: Hyperbolic limits towards macroscopic description. Math. Models Methods Appl. Sci., 17 (2007), 1675-1692.

[16] N. Bellomo, N. K. Li, P. K. Maini. On the foundations of cancer modelling: selected topics, speculations, and perspectives. Math. Models Methods Appl. Sci., 18 (2008), 593-646.

[17] A. Bertuzzi, A. Fasano, A. Gandolfi, C. Sinisgalli. ATP production and necrosis formation in a tumour spheroid model. Math. Model. Nat. Phenom., 2 (2007), 30-46.

[18] N. Bessonov, F. Crauste, I. Demin, V. Volpert. Dynamics of erythroid progenitors and erythroleukemia. Math. Model. Nat. Phenom., 4. 
[19] C. J. W. Breward, H. M. Byrne, C. E. Lewis. A multiphase model describing vascular tumour growth. Bull. Math. Biol., 65 (2003), 609-640.

[20] H. Byrne, D. Drasdo. Individual-based and continuum models of growing cell populations: a comparison. J. Math. Biol., 58 (2009), 657-687.

[21] F. A. C. C. Chalub, P. A. Markowich, B. Perthame, C. Schmeiser. Kinetic models for chemotaxis and their drift-diffusion limits. Monatsh. Math., 142 (2004), 123-141.

[22] M. A. J. Chaplain, L. Graziano, L. Preziosi. Mathematical modelling of the loss of tissue compression responsiveness and its role in solid tumour development. Math. Med. Biol., 23 (2006), 197-229.

[23] S. J. Chapman, R. J. Shipley, R. Jawad. Multiscale modeling of fluid transport in tumors. Bull. Math. Biol., 70 (2008), 2334-2357.

[24] A. Chauviere, T. Hillen, L. Preziosi. Modeling cell movement in anisotropic and heterogeneous network tissues. Netw. Heterog. Media, 2 (2007), 333-357 (electronic).

[25] J. Clairambault. Modelling physiological and pharmacological control on cell proliferation to optimise cancer treatments. Math. Model. Nat. Phenom., 4.

[26] V. Cristini, A. Li, J. S. Lowengrub, S. M. Wise. Nonlinear simulations of solid tumor growth using a mixture model: invasion and branching. J. Math. Biol., 58 (2009), 723763.

[27] M. Doumic. Analysis of a population model structured by the cells molecular content. Math. Model. Nat. Phenom., 2 (2007), 121-152.

[28] D. Drasdo. Coarse graining in simulated cell populations. Adv. Complex Syst., 8 (2005), 319.

[29] D. Drasdo, S. Hoehme. Individual-based approaches to birth and death in avascular tumors. Math. Comput. Mod., 37 (2003), 1163-1175.

[30] D. Drasdo, S. Hoehme. A single-cell-based model of tumor growth in vitro: monolayers and spheroids. Phys. Biol., 2 (2005), 133-147.

[31] S. J. Franks, J. R. King. Interactions between a uniformly proliferating tumour and its surroundings: uniform material properties. Math. Med. Biol., 20 (2003), 47-89.

[32] A. Friedman. Free boundary problems associated with multiscale tumor models. Math. Model. Nat. Phenom., 4.

[33] J. Galle, M. Loeffler, D. Drasdo. Modelling the effect of deregulated proliferation and apoptosis on the growth dynamics of epithelial cell populations in vitro. Biophys. J., 88 (2005), 62-75. 
[34] J. Galle, L. Preziosi, A. Tosin. Contact inhibition of growth described using a multiphase model and an individual cell-based model. Appl. Math. Lett. doi: 10.1016/j.aml.2008.06.051.

[35] P. Gerlee, A. R. A. Anderson. A hybrid cellular automaton model of clonal evolution in cancer: The emergence of the glycolytic phenotype. J. Theor. Biol., 250 (2007), 705-722.

[36] P. Gerlee, A. R. A. Anderson. An evolutionary hybrid cellular automaton model of solid tumour growth. J. Theor. Biol., 246 (2007), 583-603.

[37] T. Hillen. $M^{5}$ mesoscopic and macroscopic models for mesenchymal motion. J. Math. Biol., 53 (2006), 585-616.

[38] S. Hoehme, D. Drasdo. Biomechanical versus nutrient control: what determines the growth dynamics of mammalian cell populations? Submitted.

[39] Y. Jiang, J. Pjesivac-Grbovic, C. Cantrell, J. P. Freyer. A multiscale model for avascular tumor growth. Biophys. J., 89 (2005), 3884-3894.

[40] Y. Kim, M. A. Stolarska, H. G. Othmer. A hybrid model for tumor spheroid growth in vitro. I. Theoretical development and early results. Math. Models Methods Appl. Sci., 17 (2007), 1773-1798.

[41] M. Lachowicz. From microscopic to macroscopic description for generalized kinetic models. Math. Models Methods Appl. Sci., 12 (2002), 985-1005.

[42] P. Macklin, S. McDougall, A. R. A. Anderson, M. A. J. Chaplain, V. Cristini, J. Lowengrub. Multiscale modelling and nonlinear simulation of vascular tumour growth. J. Math. Biol., 58 (2009), 765-798.

[43] S. R. McDougall, A. R. A. Anderson, M. A. J. Chaplain. Mathematical modelling of dynamic adaptive tumour-induced angiogenesis: clinical implications and therapeutic targeting strategies. J. Theor. Biol., 241 (2006), 564-589.

[44] S. R. McDougall, A. R. A. Anderson, M. A. J. Chaplain, J. A. Sherratt. Mathematical modelling of flow through vascular networks: implications for tumour-induced angiogenesis and chemotherapy strategies. Bull. Math. Biol., 64 (2002), 673-702.

[45] M. R. Owen, T. Alarcón, P. K. Maini, H. M. Byrne. Angiogenesis and vascular remodelling in normal and cancerous tissues. J. Math. Biol., 58 (2009), 689-721.

[46] L. Preziosi, D. Ambrosi, C. Verdier. An elasto-visco-plastic model of cell aggregates. Submitted.

[47] L. Preziosi, A. Tosin. Multiphase modelling of tumour growth and extracellular matrix interaction: mathematical tools and applications. J. Math. Biol., 58 (2009), 625-656. 
[48] M. Radszuweit, M. Block, J. G. Hengstler, E. Schöll, D. Drasdo. Comparing the growth kinetics of cell populations in two and three dimensions. Phys. Rev. E, 79 (2009), 051907.

[49] I. Ramis-Conde, M. A. J. Chaplain, A. R. A. Anderson, D. Drasdo, P. Ghysels, G. Samaey, B. Tijskens, P. Van Liedekerke, H. Ramon, D. Roose. Multi-scale modelling of cancer cell intravasation: the role of cadherins in metastasis. Phys. Biol., 6 (2009), 12pp.

[50] I. Ramis-Conde, D. Drasdo, A. R. A. Anderson, M. A. J. Chaplain. Modeling the influence of the E-cadherin- $\beta$-catenin pathway in cancer cell invasion: A multiscale approach. Biophys. J., 95 (2008), 155-165.

[51] K. A. Rejniak. A single-cell approach in modeling the dynamics of tumor microregions. Math. Biosci. Eng., 2 (2005), 643-655.

[52] K. A. Rejniak. An immersed boundary framework for modelling the growth of individual cells: an application to the early tumour development. J. Theor. Biol., 247 (2007), 186-204.

[53] K. A. Rejniak, R. H. Dillon. A single cell-based model of the ductal tumour microarchitecture. Comp. Math. Methods Med., 8 (2007), 51-69.

[54] B. Ribba, T. Colin, S. Schnell. A multiscale mathematical model of cancer, and its use in analyzing irradiation therapies. Theor. Biol. Medical Model., 3 (2006), 7.

[55] B. Ribba, O. Saut, T. Colin, D. Bresch, E. Grenier, J. P. Boissel. A multiscale mathematical model of avascular tumor growth to investigate the therapeutic benefit of antiinvasive agents. J. Theor. Biol., 243 (2006), 532-541.

[56] T. W. Secomb, A. W. El-Kareh. A theoretical model for the elastic properties of very soft tissues. Biorheology, 38 (2001), 305-317.

[57] R. J. Shipley, G. W. Jones, R. J. Dyson, B. G. Sengers, C. L. Bailey, C. J. Catt, C. P. Please, J. Malda. Design criteria for a printed tissue engineering construct: A mathematical homogenization approach. J. Theor. Biol. doi:10.1016/j.jtbi.2009.03.037.

[58] A. Stéphanou, S. R. McDougall, A. R. A. Anderson, M. A. J. Chaplain. Mathematical modelling of flow in $2 D$ and $3 D$ vascular networks: applications to anti-angiogenic and chemotherapeutic drug strategies. Math. Comput. Modelling, 41 (2005), 1137-1156.

[59] M. Stolarska, Y. J. Kim, H. Othmer. Multiscale models of cells and tissue dynamics. Phil. Trans. Roy. Soc. A. To appear.

[60] P. Tracqui. Biophysical models of tumour growth. Rep. Prog. Phys., 72 (2009), 056701. 
[61] R. Venkatasubramanian, M. A. Henson, N. S. Forbes. Incorporating energy metabolism into a growth model of multicellular tumor spheroids. J. Theor. Biol., 242 (2006), 440453.

[62] S. M. Wise, J. S. Lowengrub, H. B. Frieboes, V. Cristini. Three-dimensional multispecies nonlinear tumor growth - I. Model and numerical method. J. Theor. Biol., 253 (2008), $524-543$.

[63] X. Zheng, S. M. Wise, V. Cristini. Nonlinear simulation of tumor necrosis, neovascularization and tissue invasion via an adaptive finite-element/level-set method. Bull. Math. Biol., 67 (2005), 211-259. 